\title{
Bilateral macular coloboma and pigmented paravenous retinochoroidal atrophy
}

\author{
Muh-Shy Chen, Chang-Hao Yang, Jen-Shang Huang
}

Department of

Ophthalmology, College

of Medicine, National

Taiwan University,

Taipei, Taiwan, ROC

M-S Chen

C-H Yang

J-S Huang

Correspondence to:

Dr Muh-Shy Chen,

Department of

Ophthalmology, College of

Medicine, National Taiwan

University, Taipei, Taiwan,

ROC.

Accepted for publication

27 September 1991

\begin{abstract}
A patient had bilateral macular coloboma with aggregations of pigment clumps located perivascularly, predominantly paravenously, and in other parts of the retina. The Toxoplasma IgG antibody was negative. The diagnosis of bilateral macular coloboma with pigmented paravenous retinochoroidal atrophy was made and seemed to be a developmental abnormality in origin.
\end{abstract}

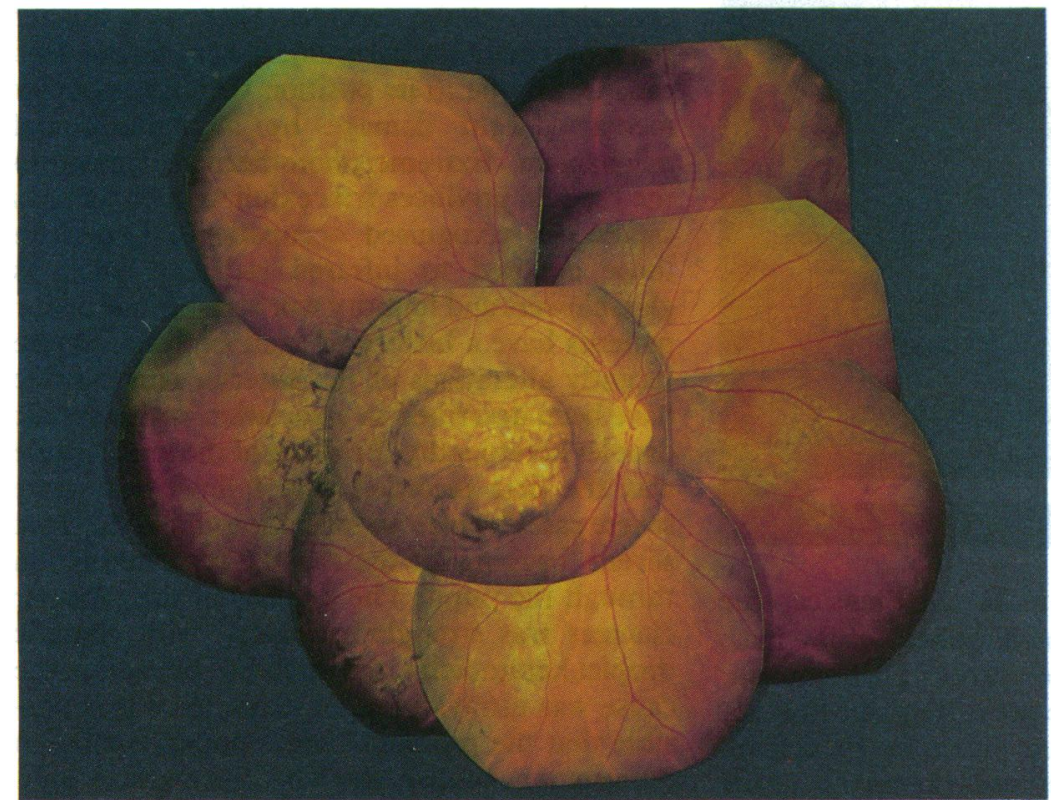

Figure 1 Retinal photograph of right fundus.

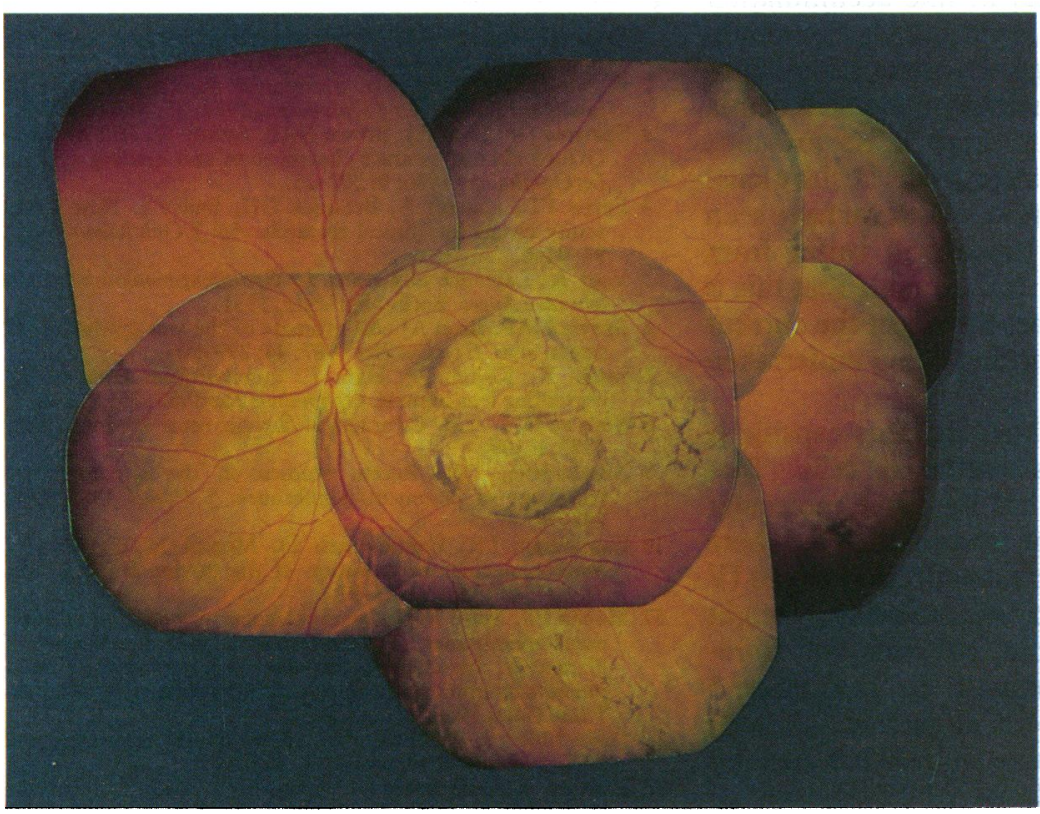

Figure 2 Retinal photograph of left fundus.
Macular coloboma is thought to result from intrauterine inflammation or as an abnormality of development. Most cases are now recognised as being due to an intrauterine infection with Toxoplasma gondii. A developmental abnormality seems to be the cause in those patients with a hereditary or family origin, ${ }^{12}$ and those with other ocular or systemic abnormalities. ${ }^{3-8}$

We report a case of bilateral macular coloboma associated with pigmented paravenous retinochoroidal atrophy and negative Toxoplasma IgG antibody in which the macular lesions seem to be of a developmental origin. To our knowledge the appearance of these two congenital anomalies has not been previously reported in the English literature.

\section{Case report}

A 23-year-old woman presented with a history of progressive blurring of vision in both eyes for a duration of 8 years. There was no contributory medical or family history.

Examination of the patient revealed the best corrected visual acuity as 20/400 in both eyes. The anterior segments were normal. Ophthalmoscopy showed a bilateral excavated, non-pigmented macular coloboma about $4 \times 4$ disc diameter in size with ectatic sclera at the base. A few large choroidal vessels were visible at the base and the retinal vessels crossing the defect were attenuated. The optic discs were normal. Aggregations of pigment clumps were located perivascularly, mainly paravenously, and in some other parts of the retina. Zones of peripapillary and radial patches of chorioretinal atrophy were situated behind the pigmented muffs and mainly along the vessels (Figs 1 and 2). Fluorescein angiography showed hypofluorescence with filling of the choroidal vasculature in both coloboma areas with a rim of hyperfluorescence surrounding the margin in the later phase of the examination. There were also blocked fluorescence from the pigment clumps and the pigment epithelial window defect due to pigment epithelial atrophy (Figs 3 and 4). The Farnsworth-Munsell 100-hue test showed a mild colour vision defect. Goldmann perimetry examination revealed central scotoma with constricted peripheral fields. Electroretinography showed a reduction in b-wave amplitude. Dark adaptation curves showed monophasic and elevated final rod thresholds in both eyes. Ultrasonography showed a bilateral ectatic base with an axial length of $21.8 \mathrm{~mm}$ in the right eye and $22.0 \mathrm{~mm}$ in the left eye.

A systemic evaluation, including skull and chest $x$ rays, complete blood count, erythrocyte sedimentation rate, antinuclear antibody, 


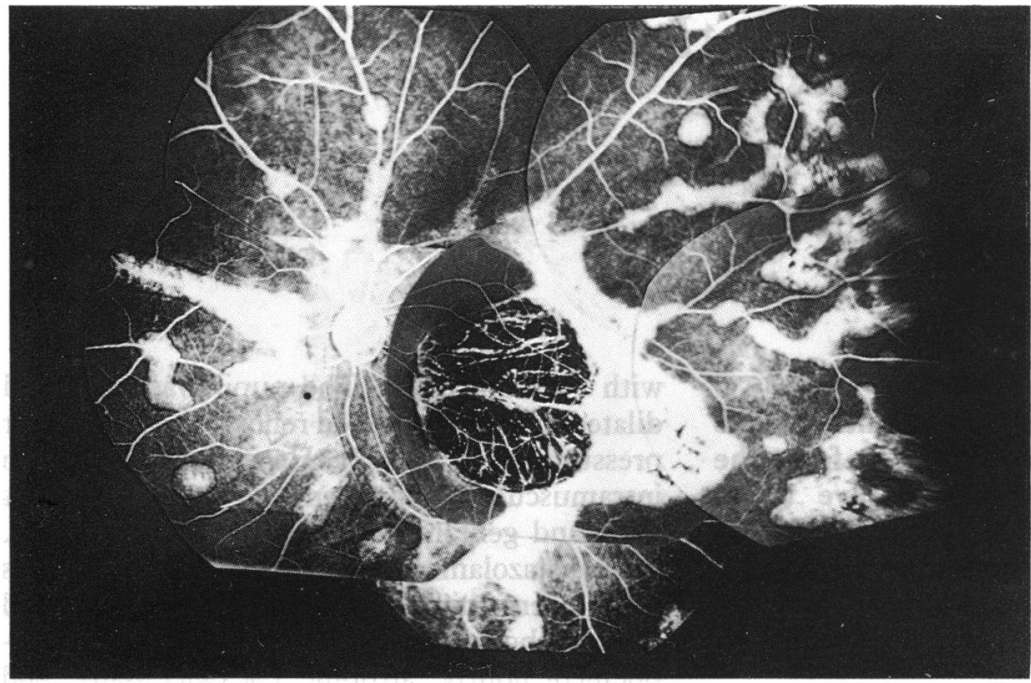

Figure 3 Fluorescein angiography of right fundus.

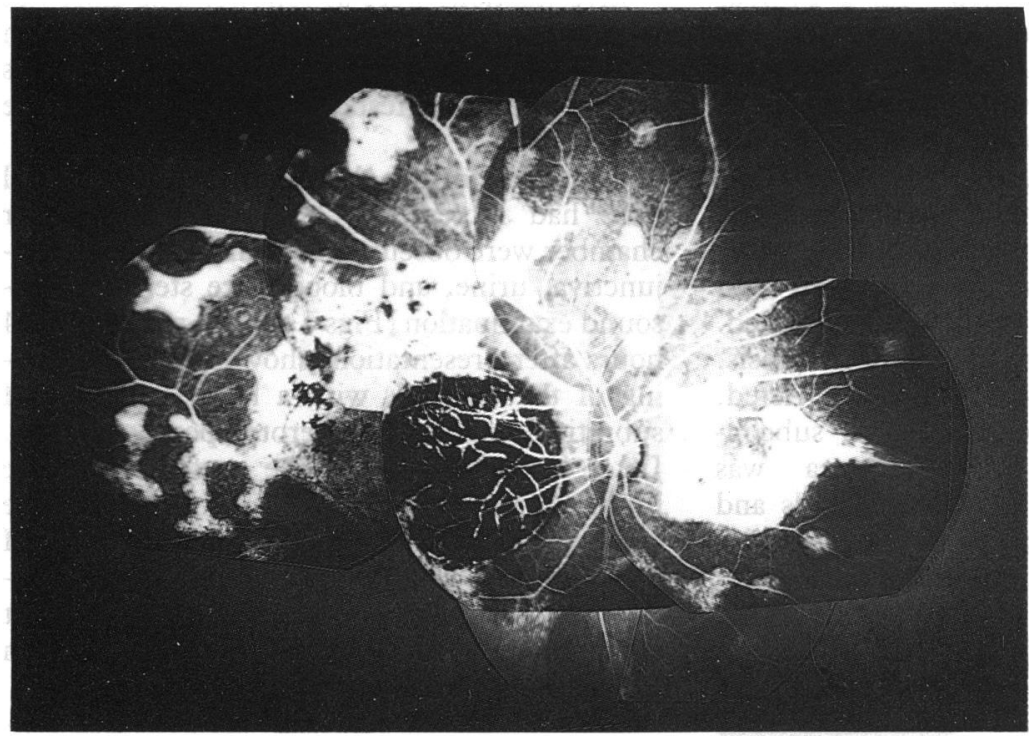

Figure 4 Fluorescein angiography of left fundus.

C-reactive protein, and VDRL test was noncontributory. Serological tests for cytomegalovirus, rubella, and herpes simplex were negative. A Toxoplasma IgG antibody test was negative at 1 in 10 dilutions by using an indirect fluorescence test.

\section{Discussion}

The patient described herein showed a bilateral macular coloboma and perivascular, mainly paravenous, aggregations of pigment clumps.

Mann' classified macular coloboma into three types, namely pigmented macular coloboma, non-pigmented macular coloboma, and macular coloboma associated with abnormal vessels. The present case did not fall clearly into any of these three types. Though the macular lesion seemed to be of the non-pigmented type paravenous pigment aggregations showed a peculiar manifestation.

Macular coloboma is known to develop from an intrauterine inflammation or as a developmental abnormality of the eye. The majority of cases are due to intrauterine Toxoplasma infection. ${ }^{10}$ However the infective process does not produce macular coloboma in families or in association with other ocular or systemic abnormalities such as retinitis pigmentosa, ${ }^{3}$ Leber's amaurosis, ${ }^{4}$ retinal dystrophy, ${ }^{5}$ idiopathic infantile hypercalciuria,${ }^{6}$ or a specific skeletal abnormality. ${ }^{78} \mathrm{An}$ indirect fluorescent test for measuring serum anti-Toxoplasma IgG titre was negative in this patient at 1 in 10 dilution. Desmonts ${ }^{11}$ emphasised the importance of calculating the ratio of the antibody titres between aqueous and serum to make the diagnosis. We did not perform anterior chamber paracentesis since we thought it was invasive and might be harmful to the patient. Though the negativity of the serum antibody test did not exclude the presence of a Toxoplasma infection the association with peculiar retinal changes in this case revealed that it was unlikely that the macular lesions were due to a previous Toxoplasma infection.

Pigmented paravenous retinochoroidal atrophy is characterised by the presence of perivascular, predominantly paravenous, aggregations of pigment clumps associated with peripapillary and radial zones of chorioretinal atrophy and is thought to be degenerative in origin. ${ }^{12-15}$ Though some parts of the retina in this patient showed chorioretinal atrophy the marked perivascular pigment aggregations and chorioretinal atrophy were similar to that of the disease.

The present case showed a bilateral macular coloboma with the perivascular existence of pigment clumps that seemed to result from a common insult affecting their developmental process and could not be explained by a single Toxoplasma macular lesion. The Toxoplasma IgG antibody test was negative in this patient. Though the aetiology of this case is still unknown the presence of a bilateral macular coloboma, pigmented paravenous retinochoroidal atrophy, and negative laboratory examinations lead us to speculate that it is a developmental abnormality in nature.

The authors thank Miss L S Yen for typing and preparing the manuscript.

1 Evans PJ. Familial macular colobomata. Br $\mathcal{F}$ Ophthalmol 1937; 21: 503-6.

2 Miller SA, Bresnick G. Familial bilateral macular colobomata. BrF Ophthalmol 1978; 62:261-4.

3 Freedman J, Gombos GM. Bilateral macular coloboma, keratoconus and retinitis pigmentosa. Ann Ophthalmol 1971; 3: toconus

4 Margolis S, Scher BM, Carr RE. Macular colobomas in Leber's congenital amaurosis. Am f Ophthalmol 1977; 83: 27-31.

5 Moore AT, Taylor DS, Harden A. Bilateral macular dysplasia ('colobomata') and congenital retinal dystrophy. $\mathrm{Br} \mathcal{F} \mathrm{Oph}$ thalmol 1985; 69: 691-9.

6 Gil-Gilberneau J, Galan A, Callis L, Rodrigo C. Infantile idiopathic hypercalciuria, high congenital myopia and atypical macular coloboma: a new oculorenal syndrome? F Pediatr Ophthalmol 1982; 19: 7-11.

7 Phillips CI, Griffiths DL. Macular coloboma and skeletal abnormality. Brf Ophthalmol 1969; 53: 346-9.

8 Sorsby A. Congenital coloboma of the macula: together with an account of the familial occurrence of bilateral macular coloboma in association with apical dystrophy of hands and coloboma in association with apical dys

9 Mann IC. On certain abnormal conditions of the macular region usually classed as colobomata. BrF Ophthalmol 1927 11: 99-116.

10 Perkins ES. Ocular toxoplasmosis. Br $\mathcal{F}$ Ophthalmol 1973; 57 . 1-17.

11 Desmonts G. Definitive serological diagnosis of ocular toxoplasmosis. Arch Ophthalmol 1966; 76: 839-51.

12 Brown TH. Retinochoroiditis radiata. Br f Ophthalmol 1937 21: 645-8.

$13 \mathrm{Chi}$ H. Retinochoroiditis radiata. Am $\mathcal{F}$ Ophthalmol 1948; 31 1485-7.

14 Chisholm IA, Dudgeon J. Pigmented paravenous retinochoroidal atrophy, helicoid retinochoroidal atrophy. Brf Ophthalmol 1973; 57: 584-7.

15 Pearlman JT, Kamin DF, Kopelow SM, Saxton J. Pigmented paravenous retinochoroidal atrophy. Am F Ophthalmol 1975 ; 80: 630-5. 